\title{
V028 ENHANCED SUBSURFACE GEOLOGICAL MODELLING THROUGH INTEGRATION OF DIPMETER STRUCTURAL AND SURFACE SEISMIC DATA
}

GIORGIO MALETTI and PAOLO BIFFI

AGIP SpA, SMES, PO Box 12069, 20100 Milan, Italy

The integration between dipmeter structural data and seismics has proven to be a powerful tool for enhancing the subsurface geological model in mature or partially explored areas.

By using "stick plots" (projection of dips on a vertical plane) the integration between these two types of data is made possible, and two objectives are achieved: improvement of the structural interpretation of dipmeter and seismics data, and the quality assessment of the processed seismic data.

Two are the main steps for dipmeter to seismics integration:

- dipmeter results filtering and reduction

- projection of selected dips on the seismic line

Dipmeter derived dips may have, as a general rule, a twofold meaning: structural and sedimentological. In fact, processing parameters normally used in a basic processing often allow to provide both types of information.

Furthermore, each computed dip and azimuth couplet is applicable only to a near-wellbore area, and may not coincide with the structural-geological trend.

The first step in dipmeter to seismics integration is thus represented by the identification of the structural-geological trend from well data.

This is achieved by performing a vertical zonation of the arrow plot in order to highlight intervals pertaining to the same dip domain (usually intervals in which the bedding attitude is constant or varies smoothly with depth, and that are constrained between sharp breaks in the value of dip and/or azimuth - i.e. main faults, unconformities, etc.). Relying on this vertical zonation, computed dip data are then filtered in order to remove dips related to sub-seismic structures, sedimentary structures and processing artefacts that are not in agreement with the structural-geological trend in each considered interval.

To be displayed on a section, filtered dip data have then to be reduced to a density compatible with seismics. In fact, an output file from a standard dipmeter processing has usually a density of 1 dip per $0.5-1 \mathrm{~m}$, whilst a single seismic reflector obviously represents a thicker interval (from $20 \mathrm{~m}$ upwards, depending on several factors). The vertical density of dips to be displayed on seismics usually ranges from 1 dip per $20 \mathrm{~m}$ to 1 dip per $50 \mathrm{~m}$.

A second critical step in dipmeter to seismics integration is represented by the projection of the reduced dip data set on the seismic section.

In fact, in the case the well trajectory doesn't lay on the plane of the seismic section, dip data have to be projected along a line parallel to the axis of the structure drilled by the well.

Moreover, since seismic sections are usually displayed in time, each selected apparent dip has to be corrected from depth to time domain. This can be achieved by using simple but reliable algorithms.

Four case histories are presented, each involving different aspects of dipmeter to seismics integration. 
Well $A$ : a dip break in the arrow plot could have been interpreted as related to an onlap, or to an erosional truncation of a previously tilted interval. The projection of the stick plot on seismic allowed to give credit to some faint and hardly visible reflectors, and to choose the second solution as the right interpretation.

Well B: this well represents an example of the improvement of dipmeter and seismics structural interpretation in case of very complex tectonical setting, and low seismic resolution due to depth $(3.8 \mathrm{~s}$ TWT). Faults interpretation from wellbore data has been matched to seismics.

Well $C$ : In this well, very high dips were found in corrispondence with the lower reservoir, where beds were expected to be almost horizontal. The display of the stick plot on seismics allowed to point out problems in the seismic migration, due to high complexity of the velocity law in the area. This resulted in a lateral shift of the seismic image of the structure, with respect to the real geographical position of the structure itself. The problem has been then partially solved by using a pre-stack depth migration processing.

Well $D$ and $D$ side-track: high angle dips belonging to a steep forward limb of the anticline encountered in well $\mathrm{D}$, are not clearly visible on the seismic line.

The integration of dipmeter data led to the drilling of a side-track in a more appropriate structural position.

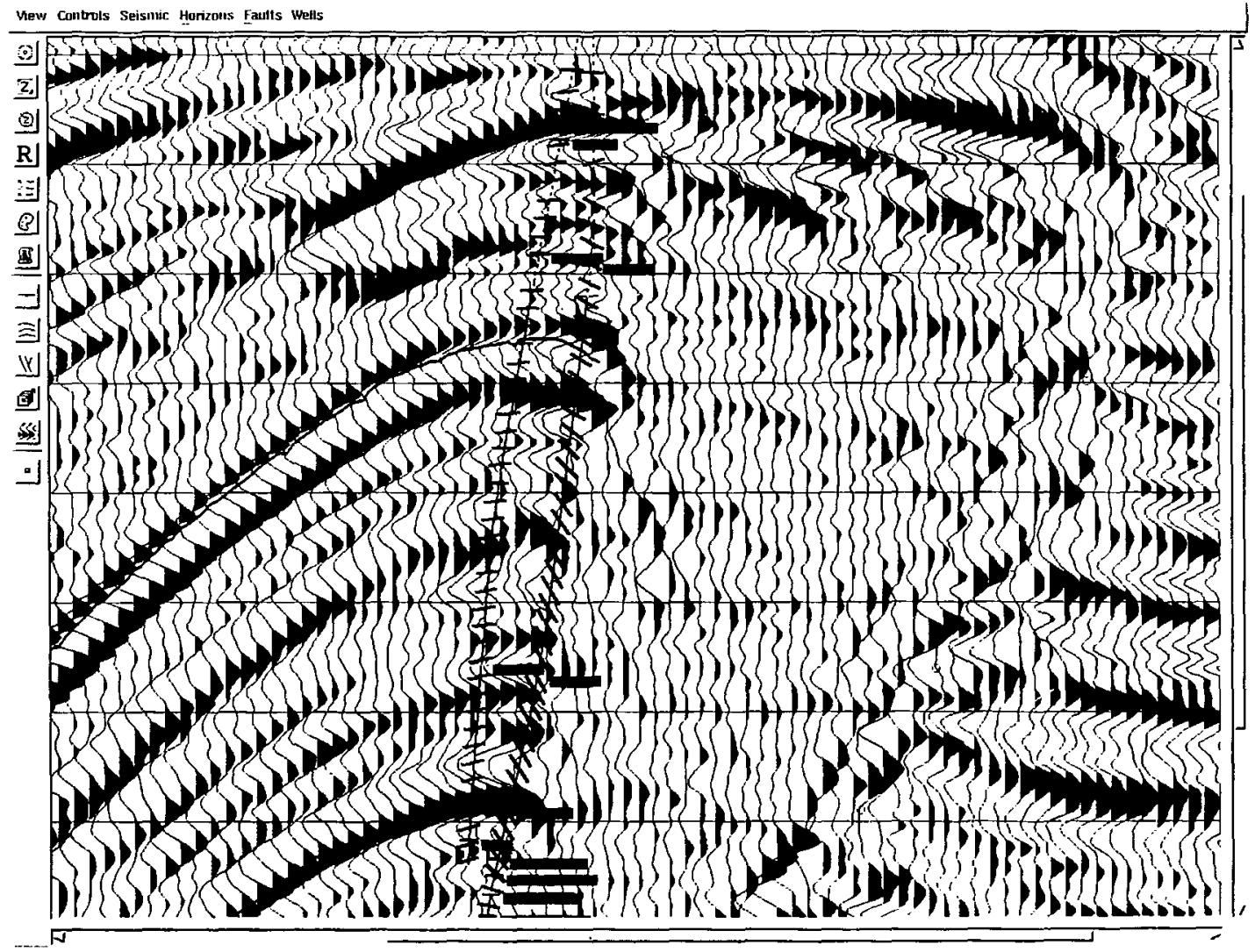

Well D (on the right) and well D side-track (on the left). 